\title{
HIDROGENAÇÃO ELETROCATALÍTICA DE SUBSTRATOS ORGÂNICOS. ESTUDO DA EFICIÊNCIA DOS METAIS NOBRES NÍQUEL, PALÁDIO E PLATINA USANDO ELETRODOS MODIFICADOS
}

\author{
José Olavo S. Pontólio, Fabiana L. S. Purgato e José R. Romero* \\ Departamento de Química, Faculdade de Filosofia, Ciências e Letras de Ribeirão Preto, Universidade de São Paulo, \\ Av. Bandeirantes, 3900, 14040-901 Ribeirão Preto - SP
}

Recebido em 2/6/03; aceito em 6/11/03; publicado na web em 27/05/04

\begin{abstract}
ELECTROCATALYTIC HYDROGENATION OF ORGANIC SUBSTRATES. STUDY OF THE EFFICIENCY OF THE NICKEL, PALADIUM AND PLATINUM NOBLE METALS USING MODIFIED ELECTRODES. Nickel, palladium and platinum microcrystals were dispersed in films covering a vitreous carbon plate electrode by ion exchange followed by electroreduction of their ions. These modified electrodes were used in the electrocatalytic hydrogenation of several substrates of different classes and their efficiency is reported. A comparison among them was performed based on the structural characteristics of the metals. A modified electrode containing platinum showed to be more efficient than a palladium modified electrode and the one of nickel was the less efficient.
\end{abstract}

Keywords: electrocatalytic hydrogenation; modified electrodes; noble metal efficiency.

\section{INTRODUÇÃO}

Reações de adição de $\mathrm{H}_{2}$ a duplas ou triplas ligações de substratos orgânicos como olefinas, alcinos, compostos carbonílicos e outras funções, estão entre as mais importantes na química orgânica preparativa. Podem ser feitas através de diferentes métodos: hidrogenação catalítica (HC) homogêneas ou heterogêneas, redução eletroquímica (RE) e hidrogenação eletrocatalítica (HEC). HC e HEC utilizam, na maior parte das vezes, um metal nobre que adsorve a espécie orgânica e o hidrogênio e este se transfere para a insaturação. Os metais nobres, de transição, são os do grupo do níquel (Ni, Pd e Pt).

A HC segue o seguinte mecanismo

$$
\begin{aligned}
& 2 \mathrm{M}+\mathrm{H}_{2} \rightleftharpoons 2 \mathrm{M}-\mathrm{H} \\
& \mathrm{M}+\mathrm{Y}=\mathrm{Z} \rightleftharpoons \mathrm{M}(\mathrm{Y}=\mathrm{Z}) \\
& \mathrm{M}(\mathrm{Y}=\mathrm{Z}+\mathrm{M}-\mathrm{H} \rightleftharpoons \mathrm{M}(\mathrm{Y}-\mathrm{ZH})+\mathrm{M} \\
& \mathrm{M}(\mathrm{Y}-\mathrm{ZH})+\mathrm{M}-\mathrm{H} \rightleftharpoons \mathrm{M}(\mathrm{YH}-\mathrm{ZH})+\mathrm{M} \\
& \mathrm{M}(\mathrm{YH}-\mathrm{ZH}) \rightleftharpoons \mathrm{M}+\mathrm{YH}-\mathrm{ZH}
\end{aligned}
$$

no qual $\mathrm{H}_{2}$ e substrato são adsorvidos, as respectivas ligações $\sigma$ e $\pi$ são quebradas e $2 \mathrm{H}$ · são transferidos em duas etapas para a molécula orgânica que sofre dessorção ${ }^{1-4}$. É necessário que o metal esteja finamente dividido porque se trata de uma reação em várias fases envolvendo sólido, líquido (solução com o substrato) e gás, sendo um fenômeno de superfície. Freqüentemente é necessário também aquecer e utilizar várias atmosferas de pressão do $\mathrm{H}_{2}$ para aumentar a velocidade da reação.

A RE pode levar aos mesmos produtos que a $\mathrm{HC}$, desde que exista uma solução de ácido ou um solvente prótico. Ocorre no cátodo uma transferência eletrônica para o sítio eletróforo do substrato com baixa densidade eletrônica, pela aplicação de potencial adequado. Este cátodo não precisa, necessariamente, ser um metal de transição. O ânion radical formado pode ser deslocalizado com separação entre o radical e o ânion. Eles podem localizar-se na molécula em sítios diferentes dependendo da sua topografia eletrônica, ficando o ânion

\footnotetext{
*e-mail: jrromero@ffclrp.usp.br
}

no sítio de mais baixa densidade. Este último pode formar uma ligação com o $\mathrm{H}^{+}$e a espécie radical resultante ser novamente reduzida formando um outro ânion que, por sua vez, reage com outro $\mathrm{H}^{+}$.

$$
\begin{aligned}
& \mathrm{R}+\mathrm{e} \rightleftharpoons \mathrm{R}^{\bullet} \longrightarrow \text { dimerização } \\
& \mathrm{R}+\mathrm{H}^{+} \rightleftharpoons \mathrm{RH}^{\cdot} \longrightarrow \text { dimerização } \\
& \mathrm{RH}^{\cdot}+\mathrm{e} \rightleftharpoons \mathrm{RH}^{-} \\
& \mathrm{RH}^{-}+\mathrm{H}^{+} \rightleftharpoons \mathrm{RH}_{2}
\end{aligned}
$$

Potenciais mais negativos são necessários para a redução de moléculas orgânicas e podem ser formados vários produtos como dímeros, a partir de dois dos radicais intermediários, competindo com o produto hidrogenado desejado ${ }^{1,5}$.

$\mathrm{Na} \mathrm{HEC}$, íons $\mathrm{H}^{+}$são reduzidos eletroquimicamente a potenciais menos negativos aplicados a uma solução de ácido mineral, formando radicais que adsorvem sobre o metal de transição juntamente com a espécie orgânica.

$$
\begin{aligned}
& \mathrm{H}^{+}+\mathrm{e} \rightleftharpoons \mathrm{H}^{\cdot} \\
& 2 \mathrm{H}^{\cdot} \longrightarrow \mathrm{H}_{2} \\
& \mathrm{H}^{\cdot}+\mathrm{M} \rightleftharpoons \mathrm{M}-\mathrm{H}
\end{aligned}
$$

Aqui também o estado de partição do metal é crucial e a partir da formação de $\mathrm{M}-\mathrm{H}$ o mecanismo é idêntico ao mostrado para a $\mathrm{HC}^{6,7}$. As vantagens da $\mathrm{HEC}$ em relação à $\mathrm{HC}$ são que o $\mathrm{H}^{*}$ é gerado in situ na superfície do eletrodo, eliminando problemas de estocagem e transporte de gás, o controle das quantidades de hidrogênio produzido pode ser feito ajustando-se o potencial ou a densidade de corrente, as condições experimentais são mais brandas (temperatura e pressão ambiente) porque o hidrogênio já é produzido sob uma forma ativada e eliminam-se problemas relacionados com envenenamento do catalisador, aplicando-se um potencial conveniente escolhido durante a eletrólise ${ }^{7}$. Observa-se no cátodo a geração de $\mathrm{H}_{2}$ na forma de bolhas que se desprendem e que pode ser formado por reação de dois radicais adsorvidos antes que reajam com a molécula orgânica também adsorvida.

Muitos métodos e materiais diferentes podem ser utilizados para as HEC e citá-los ultrapassaria em muito o objetivo deste trabalho. 
Em nosso grupo de pesquisa temos trabalhado com HEC de vários substratos orgânicos, utilizando novos filmes desenvolvidos por nós. O eletrodo de carbono vítreo foi recoberto pela eletropolimerização oxidativa dos monômeros éter alílico do ácido $p$-fenilsulfônico $(\mathrm{I})^{8} \mathrm{e}$ perclorato do éter alílico do $p$-fenil-2-etilamônio (II) ${ }^{9,10}$ (Figura 1).
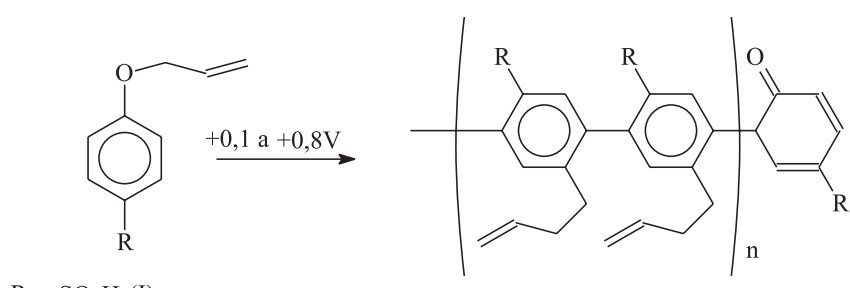

$\mathrm{R}=-\mathrm{SO}_{3} \mathrm{H}(\mathrm{I})$

$-\mathrm{CH}_{2} \mathrm{CH}_{2} \mathrm{NH}_{3}{ }^{+} \mathrm{ClO}_{4}^{-}$(II)

Figura 1. Polimerização dos éteres alílicos gerando o filme que recobre o eletrodo modificado

Os poli-éteres alílicos formados são trocadores iônicos e revestem o eletrodo com notável estabilidade mecânica e química ${ }^{8-11}$. Através de troca iônica e redução a cristalitos dos íons correspondentes aos metais nobres, foram preparados os eletrodos modificados EMs utilizados na hidrogenação eletrocatalítica de vários substratos orgânicos como compostos carbonílicos, olefinas e acetilenos ${ }^{8-10,12,13}$ (Figura 2).

$$
\begin{aligned}
& \text { poli-(I) } \stackrel{\mathrm{NiSO}_{4}}{\longrightarrow} \text { poli-(I)Ni }{ }^{2+} \stackrel{0,0 \mathrm{a}-0,9 \mathrm{~V}}{\longrightarrow} \text { poli-(I)Ni }{ }^{0} \quad \text { EM Ni } \\
& \text { poli-(II) } \stackrel{\mathrm{PdCl}_{4}^{2-}}{\longrightarrow} \text { poli-(II)PdCl}{ }_{4}^{2-} \stackrel{0,0 \mathrm{a}-0,9 \mathrm{~V}}{\longrightarrow} \text { poli-(II)Pd }{ }^{0} \quad \text { EM Pd } \\
& \text { poli-(II) } \stackrel{\mathrm{PtCl}_{4}{ }^{2-}}{\longrightarrow} \text { poli-(II) } \mathrm{PtCl}_{4}{ }^{2+} \stackrel{0,0 \mathrm{a}-0,9 \mathrm{~V}}{\longrightarrow} \text { poli-(II) } \mathrm{Pt}^{0} \quad \text { EM Pt }
\end{aligned}
$$

Figura 2. Preparação dos EMs contendo cristalitos de Ni, Pd e Pt

Por que especialmente os metais nobres se prestam à HC ou HEC?

A diminuição dos raios atômicos com o aumento do número atômico ao longo do período, moderada para os períodos 4,5 e 6 , faz diminuir o volume atômico. Nestes períodos estão localizados os metais de transição, com os quais ocorre antes o preenchimento completo do orbital s da próxima camada $(4 \mathrm{~s}, 5 \mathrm{~s}$ e $6 \mathrm{~s})$ e, em seguida, segue-se o preenchimento dos orbitais $3 \mathrm{~d}, 4 \mathrm{~d}$ e $5 \mathrm{~d}$, respectivamente ${ }^{14}$. Até a família do Co ([Ar] $\left.3 \mathrm{~d}^{7} 4 \mathrm{~s}^{2}\right)$ (...Rh e Ir), a diferença de energia entre os subníveis d e $\mathrm{s}$ respectivos impede o desemparelhamento dos elétrons dos orbitais s de valência. Este orbital mais externo, preenchido, faz com que haja uma repulsão entre seus elétrons e os elétrons do orbital $\mathrm{s}$ do $\mathrm{H}_{2}$ ou os elétrons de ligação $\pi$ de duplas ligações $\mathrm{C}=\mathrm{C}$ ou $\mathrm{C}=\mathrm{O}$, que é denominada repulsão de Pauli ${ }^{15}$. A repulsão de Pauli estabelece uma barreira frente à adsorção de $\mathrm{H}_{2}$ (maior para esta espécie devido a ter ele uma ligação s também) e de substratos orgânicos insaturados nos orbitais d da maioria dos metais de transição, impedindo que estes metais catalisem a hidrogenação das insaturações ${ }^{16}$. No entanto, os metais da família do Ni (...Pd e Pt) adsorvem $\mathrm{H}_{2}$ e insaturações ${ }^{2}$.

$\mathrm{Ni}(28 \mathrm{e}):[\mathrm{Ar}] 3 \mathrm{~d}^{8} 4 \mathrm{~s}^{2} \rightarrow \mathrm{Ni}(28 \mathrm{e}):[\mathrm{Ar}] 3 \mathrm{~d}^{9} 4 \mathrm{~s}^{1}$

$\mathrm{Pd}$ (46e): $[\mathrm{Kr}] 4 \mathrm{~d}^{10} \rightarrow \operatorname{Pd}(46 \mathrm{e}):[\mathrm{Kr}] 4 \mathrm{~d}^{9} 5 \mathrm{~s}^{1}$

Pt (78e): [Xe] $4 \mathrm{f}^{14} 5 \mathrm{~d}^{9} 6 \mathrm{~s}^{1}$

A maior diminuição do volume atômico nesta família aproxima mais os subníveis de energia d e s que os outros metais de transição mais à esquerda e torna possível a re-hibridização do Ni e Pd mostrada nas equações acima, onde os elétrons ficam desemparelhados ${ }^{15}$.
A energia do choque entre as espécies que serão adsorvidas com os átomos expostos na camada externa da superfície do metal é, agora, suficiente para que ocorra esta re-hibridização ${ }^{16}$. Assim, estes orbitais externos desemparelhados do Ni, Pd e Pt podem aceitar os elétrons do H· e C.-C· formando ligações relativamente estáveis, em um processo conhecido como quimiossorção $0^{2,3,16}$.

O Ni tem um arranjo cristalográfico mais compactado devido a um menor volume (conseqüentemente, um menor número de faces expostas para a adsorção) $)^{16}$.

$\mathrm{O}$ paládio apresenta absorção de $\mathrm{H}_{2}$ e de moléculas orgânicas ${ }^{4}$ além do fenômeno da adsorção.

A platina já se apresenta com os elétrons desemparelhados, diferentemente do metal $\mathrm{Ir}\left([\mathrm{Xe}] 4 \mathrm{f}^{14} 5 \mathrm{~d}^{7} 6 \mathrm{~s}^{2}\right)$ que ela segue no período ${ }^{14}$.

$\mathrm{O}$ presente trabalho visa realizar a comparação entre os resultados obtidos em HEC de várias classes de substratos orgânicos, utilizando cristalitos de $\mathrm{Ni}$, Pd e Pt dispersos em uma matriz polimérica recobrindo eletrodo de carbono vítreo.

\section{PARTE EXPERIMENTAL}

\section{Equipamentos}

Os experimentos eletroquímicos foram realizados nos aparelhos Potenciostato/Galvanostato PAR modelo 273A, com a aquisição de dados realizada com o programa Model 270 Electrochemical Analysis Software e no Potenciostato MQPG - 01, com a aquisição dos dados realizada pelo programa MQPG.

As voltametrias cíclicas para a preparação do filme e a redução dos sais aos cristalitos de seus metais utilizaram placa de carbono vítreo $(\mathrm{CV})^{17}$ de 2,6 x 4,5 x 0,15 cm de espessura (área de 23,4 $\mathrm{cm}^{2}$ ) como eletrodo de trabalho, em cela cilíndrica de $100 \mathrm{~mL}$ de capacidade. Este eletrodo foi colocado de forma simétrica no centro de uma rede de platina com $1,0 \mathrm{~cm}$ de altura e $80 \mathrm{~cm}$ de comprimento e $1 \mathrm{~mm}$ de espessura, com área de $164 \mathrm{~cm}^{2}$, enrolada sobre si mesma como num cilindro e presa por um fio de platina. Esta rede foi usada como eletrodo auxiliar e como eletrodo de referência foi utilizado o eletrodo de $\mathrm{Ag}^{0} / \mathrm{AgCl}^{10}$.

Após a deposição do filme por oxidação e polimerização do monômero, a placa de $\mathrm{CV}$ apresentou $19 \mathrm{~cm}^{2}$ de área superficial recoberta.

Para as HEC, usando-se a placa de CV, a rede de platina foi substituída por um fio de platina dentro de um tubo de vidro com fundo de vidro sinterizado.

Os produtos das HEC foram identificados e seus rendimentos calculados após extração com $\mathrm{CH}_{2} \mathrm{Cl}_{2}$, secagem e evaporação do solvente. Métodos espectroscópicos convencionais foram utilizados. Os espectros de ressonância magnética nuclear ${ }^{1} \mathrm{H}$ RMN foram obtidos em um aparelho Bruker modelo Avance DRX 400 (400 MHz). Os deslocamentos químicos $(\delta)$ referem-se ao padrão interno (TMS) registrado em parte por milhão.

Os espectros de absorção no infravermelho (IV) foram registrados em um espectrômetro Perkin Elmer modelo RX Series FTIR System. Utilizou-se filme líquido ou pastilhas de $\mathrm{KBr}$.

Quando se dispunha de uma amostra igual ao produto obtido, a comparação foi feita por cromatografia a gás. As análises por cromatografia gasosa (CG) foram efetuadas em um cromatógrafo Intralab modelo 3400 com coluna OV-17 e um Integrador Intralab modelo 4290, utilizando-se detector de ionização de chama.

Quando o substrato absorvia na faixa correspondente ao UVVIS (a maioria deles), seu consumo na HEC foi acompanhado pela espectroscopia correspondente.

Os estudos espectroscópicos UV-VIS foram realizados em cubetas de quartzo de $1 \mathrm{~cm}$ de caminho óptico e os registros foram 
feitos em um espectofotômetro HP modelo 8453 com controlador de temperatura HP modelo 89090A acoplado. O espectrofotômetro foi acoplado a um computador HP Vectra XA.

Os eletrodos estudados tiveram seus filmes removidos por polimento usando-se o kit de polimento PK-4 da BAS - Bioanalytical Systems Inc.

\section{Preparação dos eletrodos modificados}

Revestimento do eletrodo pelo filme poli-(éter alílico do p-benzenossulfonato de sódio $)^{8}$

Em uma solução contendo éter alílico do $p$-benzenossulfonato de sódio dissolvido em $\mathrm{H}_{2} \mathrm{SO}_{4} 0,5 \mathrm{~mol} \mathrm{~L}^{-1}$ foram feitas varreduras em um intervalo de potencial de $+1,0$ a $+2,0 \mathrm{~V}$ vs. $\mathrm{Ag}^{0} / \mathrm{AgCl}$, com agitação entre cada varredura, para recobrir a superfície do eletrodo de trabalho. Foi utilizada uma velocidade de varredura de $10 \mathrm{mV} \mathrm{s}^{-1}$.

\section{Incorporação do $\mathrm{Ni}^{2+}$ ao filme poli-(I) por troca iônica}

Após a preparação do filme polimérico, o eletrodo de trabalho ficou em imersão durante $2 \mathrm{~h}$ em $100 \mathrm{~mL}$ de $\mathrm{NiSO}_{4} 0,5 \mathrm{~mol} \mathrm{~L}^{-1}$ para a troca iônica dos íons $\mathrm{H}^{+}$pelos íons $\mathrm{Ni}^{2+}$ da solução.

\section{Redução do $\mathrm{Ni}^{2+}$ incorporado ao filme. Preparação do EM Ni}

Foi feita a redução dos íons $\mathrm{Ni}^{2+}$ a cristalitos metálicos através de voltametria linear em $50 \mathrm{~mL}$ de solução de $\mathrm{KCl} \mathrm{0,1} \mathrm{mol} \mathrm{L-1}$. Usou-se uma velocidade de varredura de $25 \mathrm{mV} \mathrm{s}^{-1} \mathrm{em}$ um intervalo de potencial de 0 a $-1,0 \mathrm{~V}$ vs. $\mathrm{Ag}^{0} / \mathrm{AgCl}$, de modo a obter somente a curva de redução. Várias varreduras foram feitas até não mais aparecer corrente de redução do $\mathrm{Ni}^{2+}$.

\section{Incorporação do íon tetracloropaladato ao filme poli-(II) por troca iônica}

O eletrodo formado pelo poli-[éter alil p-(2-etilamônio) benzeno $]^{9,10}$ foi imerso previamente em $30 \mathrm{~mL}$ de solução aquosa $0,5 \mathrm{~mol} \mathrm{~L}^{-1}$ de $\mathrm{KCl}$ por $30 \mathrm{~min}$. Após a incorporação do íon cloreto ao filme, o eletrodo foi imerso em $30 \mathrm{~mL}$ de solução aquosa $0,01 \mathrm{~mol} \mathrm{~L}^{-1}(125 \mathrm{mg})$ de tetracloropaladato de potássio por $30 \mathrm{~min}$.

\section{Redução do $\mathrm{PdCl}_{4}^{2-}$ incorporado ao filme. Preparação do EM Pd}

Os eletrodos contendo os íons tetracloropaladato foram submetidos a varreduras numa cela contendo $70 \mathrm{~mL}$ de solução aquosa $0,10 \mathrm{~mol} \mathrm{~L}^{-1} \mathrm{de} \mathrm{KCl}$, em um intervalo de potencial de 0 a $-1,0 \mathrm{~V}$ vs. $\mathrm{Ag}^{0} / \mathrm{AgCl}$ a $10 \mathrm{mV} \mathrm{s}^{-1}$.

\section{Preparação do Pd Ni/Pd por "electroless"18}

Dissolveram-se $0,1 \mathrm{~g}$ de $\mathrm{PdCl}_{2}$ em $10 \mathrm{~mL}$ de água destilada com agitação durante alguns minutos. Adicionaram-se 0,256 g de EDTA e a solução foi mantida à quente $\left(70{ }^{\circ} \mathrm{C}\right)$. Em seguida, adicionaramse $0,19 \mathrm{~g}$ de oxalato de sódio e potássio, $\mathrm{KNaC}_{4} \mathrm{H}_{4} \mathrm{O}_{6} \cdot 4 \mathrm{H}_{2} \mathrm{O}$. Depois se adicionou $0,04 \mathrm{~g}$ de $\mathrm{NaH}_{2} \mathrm{PO}_{2}$ e $0,07 \mathrm{~mL}$ de $\mathrm{HCl}$ concentrado. Manteve-se a solução à quente $\mathrm{e}$, sem parar a agitação, mergulhouse o EM Ni nesta solução deixando-o imerso por $5 \mathrm{~h}$. Algumas vezes o becker em que este procedimento foi realizado ficou espelhado.

\section{Preparação do eletrodo modificado EM Pt}

Os procedimentos necessários para esta preparação estão descritos na literatura ${ }^{9,10}$.
Cálculo das massas de metal e densidades de corrente na geração de $\mathrm{H}_{2}$ dos EM

As massas de cristalito de metal dispersos nos filmes I e II foram calculadas aplicando-se a lei de Faraday para a reação de redução eletroquímica dos íons incorporados nos filmes. Considerou-se o envolvimento de dois elétrons e o número de Coulombs contidos na área correspondente à descarga desta redução ${ }^{9,10}$.

A densidade de corrente da geração de $\mathrm{H}_{2}$ de uma solução ácida foi calculada pela corrente a $-1,0 \mathrm{~V}$ em função da área recoberta pelo filme no $\mathrm{EM}^{8-10}$.

\section{Hidrogenação eletrocatalítica dos substratos orgânicos}

A metodologia descrita a seguir foi idêntica para todos os eletrodos utilizados e substratos estudados.

A HEC foi feita a partir de uma solução $\left(0,02 \mathrm{~mol} \mathrm{~L}^{-1}\right)$ de substrato em $\mathrm{H}_{2} \mathrm{SO}_{4} 0,1 \mathrm{~mol} \mathrm{~L}^{-1}$. Aplicou-se um potencial controlado de $-0,90 \mathrm{~V}$ vs. $\mathrm{Ag}^{0} / \mathrm{AgCl}$, sob forte agitação magnética, durante um período de 24 h. Os substratos estudados foram benzaldeído, acetofenona, 2cicloexen-1-ona, isoforona, cicloexanona, $n$-valeraldeído, 3-butin1-ol e fenilacetileno (Tabela 2). Observou-se despreendimento de $\mathrm{H}_{2}$. A HEC foi deixada por $24 \mathrm{~h}$. O meio reacional foi neutralizado com $\mathrm{NaHCO}_{3}$ e os produtos orgânicos foram extraídos com $\mathrm{CH}_{2} \mathrm{Cl}_{2}$; a solução foi seca com $\mathrm{MgSO}_{4}$ anidro e o solvente foi evaporado. Após o isolamento, o produto bruto ou purificado por coluna cromatográfica de sílica-gel foi pesado e analisado por cromatografia a gás e métodos espectroscópicos convencionais através dos equipamentos mencionados acima.

\section{RESULTADOS E DISCUSSÃO}

A preparação destes eletrodos com os diferentes metais nobres consistiu em mergulhar o carbono vítreo revestido com os filmes poli-(I) e poli-(II) numa solução dos sais $\mathrm{NiSO}_{4}{ }^{12}, \mathrm{~K}_{2} \mathrm{PdCl}_{4}{ }^{13} \mathrm{e}$ $\mathrm{K}_{2} \mathrm{PtCl}_{4}^{9,10} \mathrm{e}$, após a troca iônica, os mesmos foram reduzidos a seus metais como cristalitos, que ficaram dispersos na matriz polimérica produzindo os eletrodos modificados EM Ni, EM Pd e EM Pt (Figura 2). Foi feito também um revestimento "electroless" ${ }^{18-20}$ com Pd (utilizando-se $\mathrm{PdCl}_{2}$ ) no EM Ni produzindo o EM Ni/Pd.

Métodos coulométricos foram utilizados para calcular a massa dos cristalitos no eletrodo e para medir a densidade de corrente dos EMs na geração de $\mathrm{H}_{2}$ numa solução ácida ${ }^{9,10}$.

A Tabela 1 mostra as massas dos cristalitos de Ni, Pd e Pt por unidade de área nos três eletrodos assinalados, bem como as densidades de corrente da geração de $\mathrm{H}_{2}$ de uma solução ácida para os quatro eletrodos. Não foi possível calcular a massa de Pd depositado por "electroless" no EM Ni.

Tabela 1. Massas dos metais e densidade de corrente para a geração de $\mathrm{H}_{2}$ dos EM

\begin{tabular}{lll}
\hline $\begin{array}{l}\text { Eletrodos } \\
\text { modificados }\end{array}$ & $\begin{array}{l}\text { Massas de metal } \\
\left(\mathrm{g} \mathrm{cm}^{-2}\right)\end{array}$ & $\begin{array}{l}\text { Densidade de } \\
\text { corrente }\left(\mathrm{mA} \mathrm{cm}^{-2}\right)\end{array}$ \\
\hline EM Ni & $1,26.10^{-7}$ & 6,93 \\
EM Pd & $5,0.10^{-7}$ & 19,2 \\
EM Pt & $4,4.10^{-6}$ & 14,1 \\
EM Ni/Pd & - & 34,8 \\
\hline
\end{tabular}

Como pode ser visto, as trocas iônicas e a redução dos íons aos cristalitos dos metais foram progressivamente mais eficientes do $\mathrm{Ni}$ até a Pt, tendo 4 vezes mais massa de $\mathrm{Pd}$ que Ni e 35 vezes mais 
massa de Pt que Ni dispersa nos filmes. Esta diferença pode ser atribuída aos diferentes monômeros utilizados para a formação dos filmes e aos diferentes íons para a troca iônica. Porém, as densidades de corrente para a redução do $\mathrm{H}^{+}$não variam muito mais que 2 vezes nos EM Pd e EM Pt para o EM Ni. Ou seja, uma massa 35 vezes maior de Pt em relação ao Ni gera apenas 2,03 vezes mais $\mathrm{H}^{*}$. A Tabela 2, que será analisada a seguir, mostra significativos maiores rendimentos na HEC da Pt com relação aos demais eletrodos. Portanto, possíveis diferenças no número ou no tamanho dos cristalitos não exerce, no presente caso, um fator decisivo. Há, para a Pt, uma maior eficácia na quimiossorção do $\mathrm{H}^{*}$ que bloqueia os centros ativos do metal para posteriores reações da redução do $\mathrm{H}^{+}$, daí uma densidade de corrente apenas 2 vezes maior. Assim, uma maior eficiência na adsorção do $\mathrm{H}^{*}$ e das ligações $\pi$ dos substratos orgânicos conduz a uma maior eficiência na reação de hidrogenação. O EM $\mathrm{Ni} / \mathrm{Pd}$ deu uma densidade de corrente aproximadamente o dobro em relação aos EM Pd e EM Pt, o que significa, à luz das considerações que foram feitas, uma eficiência menor na adsorção de $\mathrm{H}^{*}$.

Os substratos hidrogenados foram carbonílicos (aldeídos e cetonas aromáticas e alifáticas), cetona insaturada (redução da dupla ligação) e alcinos (aromáticos e alifáticos).
A Tabela 2 apresenta os resultados e os rendimentos das HECs efetuadas sempre pelo mesmo tempo $(24 \mathrm{~h})$, cujos produtos foram extraídos e caracterizados utilizando a mesma metodologia.

Nem todos os substratos estudados reagiram com todos os EMs, mas os produtos obtidos foram sempre os mesmos. O EM Pt reagiu com todos eles dando os melhores rendimentos. Benzaldeído e acetofenona foram hidrogenados a seus álcoois correspondentes com ótimos rendimentos. O fenilacetileno teve a tripla hidrogenada dando etilbenzeno também com bons rendimentos. Este desempenho superior dos aromáticos pode ser explicado pelo fato de se poder prever maior adsorção na superfície dos cristalitos de $\mathrm{Ni}, \mathrm{Pd}$ ou $\mathrm{Pt}$, devido à extensão da conjugação das carbonilas e da tripla ligação no anel aromático.

Para o EM Ni, o menos eficiente dos materiais estudados, a 2cicloexen-1-ona, uma cetona conjugada, reagiu com rendimento moderado. Já a cicloexanona, sem conjugação, deu um baixo rendimento em cicloexanol. Comparando-se os acetilenos entre si, o 3butin-1-ol não reagiu com o EM Ni. Embora o fator de maior adsorção graças à extensão de conjugação fique evidente por estes resultados, o impedimento estérico dificulta a adsorção, pois a isoforona, com uma conjugação igual à da 2-cicloexen-1-ona, foi o menos reativo

Tabela 2. Produtos e rendimentos porcentuais das HECs utilizando os EM Ni, EM Pd, EM Ni/Pd e EM Pt

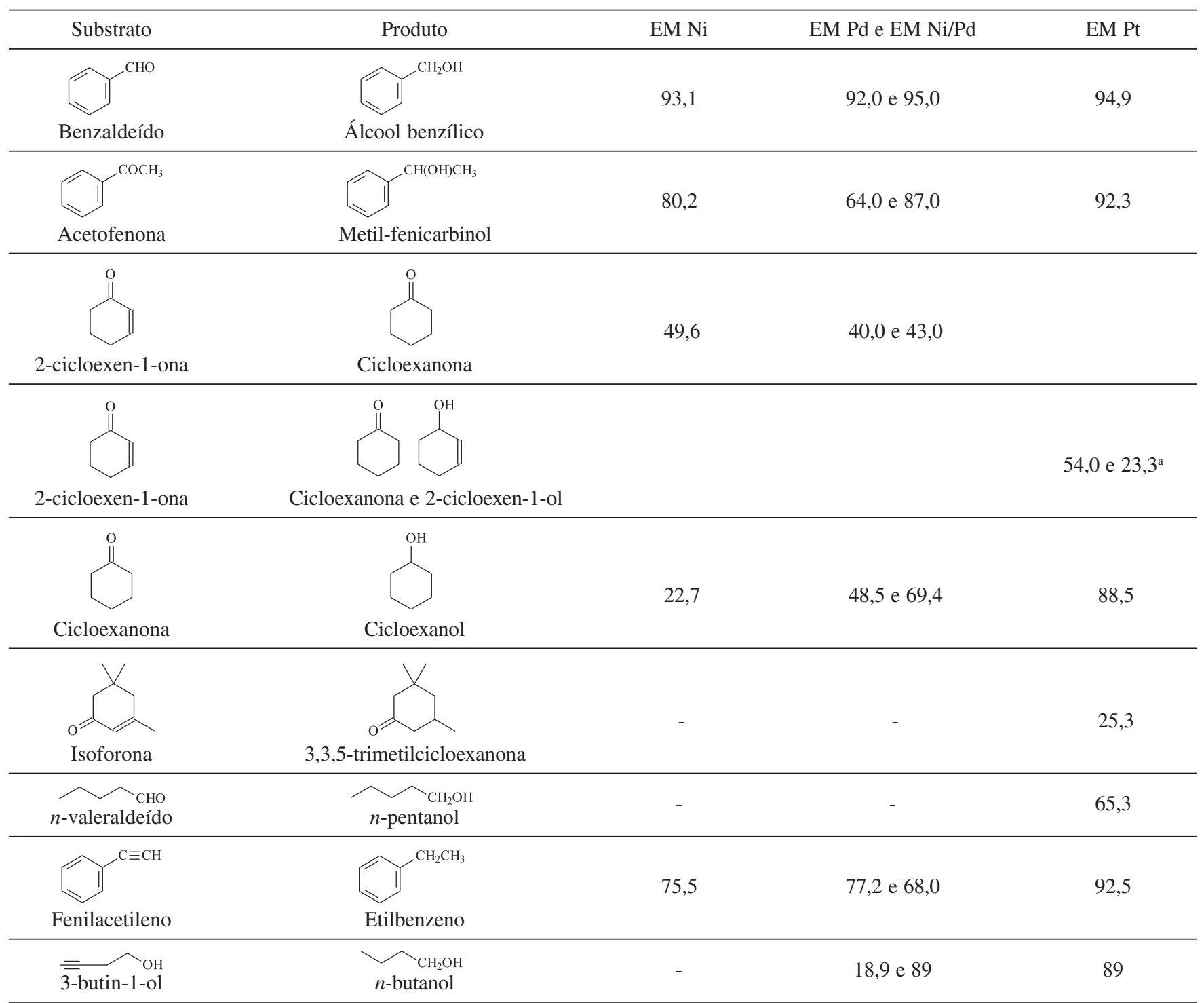

\footnotetext{
${ }^{a}$ Um dos produtos utilizando EM Pt foi diferente dos obtidos pelos demais EMs.
} 
de todos os substratos estudados, dando um rendimento pequeno apenas com o EM Pt. Este baixo desempenho deve-se ao maior impedimento estérico imposto pelas três metilas, dando o mesmo produto de hidrogenação da dupla ligação. Também o $n$-valeraldeído foi hidrogenado dando $n$-pentanol somente pelo EM Pt, com um rendimento apenas razoável. A ausência de conjugação neste aldeído saturado responde por este desempenho medíocre e reforça a interpretação da importância da conjugação citada acima.

A 2-cicloexen-1-ona teve a hidrogenação de sua dupla ligação dando cicloexanona com todos os EMs, porém obteve-se também o 2-cicloexen-1-ol utilizando o EM Pt. No entanto, quando se utilizou cicloexanona o produto obtido foi o cicloexanol. A não hidrogenação do produto cicloexanona nos resultados mencionados acima se deve a problemas de adsorção, que ainda estão sendo investigados em nossos laboratórios.

A diferença de desempenho a favor do EM Pt se comparado com o EM Ni é evidente e concorda com as premissas estruturais alinhadas na introdução. Os resultados para o Pd são mais complicados para serem explicados. O EM Pd tem uma eficiência comparável ao EM Ni, mostrando-se tão sensível quanto este com respeito à falta de extensão de conjugação para favorecer a adsorção e aos efeitos estéricos, como na ausência de produtos para a HEC do $n$-valeraldeído e da isoforona. Uma hipótese para este baixo desempenho pode ser o fato de que este metal nobre tem uma estrutura cristalográfica que permite também a absorção de $\mathrm{H}_{2}{ }^{4}$. Limitações na transferência de massa para o etileno foram observadas ${ }^{21,22}$.

O EM Ni/Pd coloca-se claramente com uma eficiência intermediária, não tendo ainda promovido a hidrogenação da isoforona e do $n$-valeraldeído, mas dando melhores rendimentos para os demais substratos, especialmente para a hidrogenação do 3-butin-1-ol. A preparação deste eletrodo visou contornar o problema da absorção. O revestimento "electroless" do EM Ni com Pd deve ter revestido o Ni com uma fina camada de Pd, não permitindo a absorção de hidrogênio ou moléculas dos substratos e não causando problemas de transferência de massa para os substratos que foram mais eficientemente adsorvidos. A alta densidade de corrente na redução de $\mathrm{H}^{+}$mostra que este desempenho se limita, em grande parte a esta reação com subseqüente formação de $\mathrm{H}_{2}$, não ocorrendo uma adsorção mais forte de $\mathrm{H}$ - como ocorre com a Pt.

\section{CONCLUSÃO}

As HEC dos substratos orgânicos aqui estudados mostraram que o EM Pt foi mais eficiente que os EMs com Ni ou Pd. A HEC de alguns substratos com diferentes funcionalidades pode, no entanto, ser efetuada pelo EM Ni, que tem um custo muito mais baixo que os outros.

A HEC mostrou-se mais produtiva para substratos que podem ser adsorvidos mais eficientemente devido à sua extensão de conjugação e que não apresentam um forte impedimento estérico.

O EM Pd apresentou problemas de eficiência que associamos com a possibilidade de absorção e conseqüentes dificuldades de transferência de massa. O revestimento "electroless" do EM Ni contornou esta dificuldade melhorando o desempenho do Pd, criando uma superfície fina sobre o cristalito de $\mathrm{Ni}$, impedindo a adsorção.

\section{AGRADECIMENTOS}

À FAPESP e ao CNPq pelas bolsas e suporte financeiro. Agradecemos a colaboração da Profa. L. M. Abrantes do Departamento de Química e Bioquímica, Faculdade de Ciências da Universidade de Lisboa, através da Cooperação Internacional CAPES/GRICES.

\section{REFERÊNCIAS}

1. Chapuzet, J. M.; Lasia, A.; Lessard, J. Em Electrocatalytic Hydrogenation of Organic Compounds in Electrocatalysis-Frontiers in Electrochemistry; Lipkowski, J.; Ross, P. N., eds.; Wiley $2^{\text {nd }}$ ed., 1998, p. 155.

2. Ibidem ref. 1, p. 168.

3. Ibidem ref. 1 , p. 159.

4. Ibidem ref. 1, p. 170.

5. Lund, H.; Baizer, M. M., eds. Em Organic Electrochemistry, an introduction and a guide; Marcel Deckker Inc., 1991, p. 505.

6. Deronzier, A.; Moutet, J. C.; Acc. Chem. Res. 1989, 22, 249.

7. Coche, L.; Moutet, J. C.; J. Am. Chem. Soc. 1987, 109, 6887.

8. Lofrano, R. C. Z.; Madurro, J. M.; Romero, J. R.; J. Mol. Catal. A: Chem. 2000, 153, 237.

9. Lofrano, R. C. Z.; Queiroz, J. V.; Romero, J. R.; J. Mol. Catal. A: Chem. 2001, 174, 231.

10. Lofrano, R. C. Z.; Padovan, N. A.; Romero, J. R.; Quim. Nova 2002, 25, 1186.

11. Madurro, J. M.; Paiva, A. P.; Romero, J. R.; Abrantes, L. M.; $13^{\circ}$ Simpósio Brasileiro de Eletroquímica e Eletroanalítica-SIBEE, Araraquara, Brasil, 2002.

12. Pontólio, J. O. S.; Romero, J. R.; $13^{\circ}$ Simpósio Brasileiro de Eletroquímica e Eletroanalítica-SIBEE, Araraquara, Brasil, 2002.

13. Purgato, F. L. S.; Romero, J. R.; $13^{\circ}$ Simpósio Brasileiro de Eletroquímica e Eletroanalítica-SIBEE, Araraquara, Brasil, 2002.

14. Russell, J. B. Em Química Geral; 2a ed., 1994, p. 308.

15. Christmann, K. Em Hydrogen Adsorption at Metal Surfaces in Electrocatalysis-Frontiers in Electrochemistry; Lipkowski, J.; Ross, P. N., eds.; Wiley, $2^{\text {nd }}$ ed., 1998, p. 15.

16. Ibidem ref. 18 , p. 13 .

17. Agradecemos a doação das placas de carbono vítreo a A. Deronzier, Laboratoire d'Electrochimie Organique, Universitè Fourrier, Grenoble.

18. Henry, J. R. Em Electroless (Autocatalytic) Plating in Standards and Guidelines for Electroplated Plastics; Metal Finishing $4^{\text {th }}$ ed., 1998, p. 431.

19. Abrantes, L. M.; Correia, J. P.; J. Electrochem. Soc. 1994, 141, 2356.

20. Correia, J. P.; Ricardo, A. P.; Abrantes, L. M.; Boletim da Sociedade Portuguesa de Química 1990, 41, 27.

21. Harris, J.; Appl. Phys. A: Mater. Sci. Process 1988, 47, 63.

22. Skellaropoulos, G. P.; Langer, S. H.; J. Catal. 1981, 67, 77. 